\title{
Multiuser Detection using Zero Forcing Successive Interference Cancellation for WCDMA
}

\author{
D K Patidar \\ Department of Electronics and \\ Communication \\ Dr.B.R. Ambedkar NIT Jalandhar \\ Punjab, India
}

\author{
H P Singh \\ Department of Physics \\ Dr.B.R.Ambedkar NIT Jalandhar \\ Punjab, India
}

\author{
S A Khan \\ Department of Electronics and \\ Communication \\ Shiv Shankar Institute of \\ Technology Patti, Punjab, India
}

\begin{abstract}
The performance of the WCDMA system is degraded due to the various network impairments such as interference, fading $\&$ noise and also due to network load. The work in this presents performance analysis of zero forcing successive interference cancellation (ZF-SIC) scheme for the multiuser detection in the WCDMA environment. The bit-error rate (BER) of WCDMA using ZF-SIC for binary phase shift keying (BPSK) and quadrature phase shift keying (QPSK) has been analyzed. The ZF-SIC scheme subtracts the strongest received signal from the original one by one till all users have been detected and demodulated. The proposed scheme also suppressed multiple-access interference (MAI) and improved the system performance significantly. The simulation results showed that the BER is decreased the with number of users increased
\end{abstract}

\section{Keywords}

Multiuser Detection, ZF-SIC, WCDMA, Interference, MAI

\section{INTRODUCTION}

In WCDMA, every communicator will be allocated the entire spectrum all of the time. It uses codes to identify connection. A conventional DS/CDMA system treats each user separately as a signal, with other users considered as noise MAI [1]. All users interfere with all other users and the interferences add to cause performance degradation. The near/far problem is serious and tight power control, with attendant complexity is needed to combat it [2]. All users in a CDMA system interfere with each other. Potentially significant capacity increases and near/far resistance can theoretically be achieved if the negative effect that each user has on others can be canceled [3]. A solution to the shortcomings of the conventional CDMA system is multiuser detection in which all users are considered as signals to each other [4]. If a higher data rate is used, the performance is degraded in a multi-user environment. Multi-user detectors take advantage of the information about the other users and hence provide better performance [5]. The optimal (maximum-likelihood) multiuser detector was discussed in [6]. However, the optimal receiver is too complex to be implemented. As complexity increased as its complexity grows exponentially with the number of users and number of detecting symbols. Suboptimal receivers trade some of this optimality to much lower complexity. The suboptimal detectors are multistage detectors; decision feedback detector, successive interference cancellation (SIC) and parallel interference cancellation (PIC) are used to mitigate the MAI [7]. The multistage detectors and decision feedback detector are decision-directed. They utilized previously made by decision of other users to cancel the interference present in the signal of the desired user. The SIC can use soft decision to mitigate the MAI. The SIC which required ordering of users according to their powers. The signal of stronger users are demodulated first and canceled from the signal of weaker users. This technique provides an efficient and practical solution to the near/far problem [8]. The Section 2 describes the multiuser detection using SIC and Section 3 presents methodology adopted for the simulations. The simulation results and discussion are presented in Section 4. The last section presents the conclusion

\section{MUD SUCCESSIVE INTEREFERENCE}

\section{CANCELLATION}

The SIC is serially cancelling the interfering users from the outputs of the matched filter in order of signal strength. Received continuous-time signal can be written as:

$r(t)=\sum_{k=1}^{K} A_{k}(t) c_{k}(t) b_{k}(t)+n$

Where the users have been ranked 1 to $\mathrm{K}$ according to increasing received signal strength. The SIC detector is easy to implement and less complex than PIC

Where $K$ is the number of users, $A_{k}(t)$ is the amplitude of user $k, c_{k}(t)$ is the code sequence and $b_{k}(t)$ is the bit sequence of user $k$ and $n(t)$ is noise. Output of the correlator of user $k$ yields $\left(\rho_{i, k}\right.$ is correlation between codes $c_{i}$ and $c_{k}$ ).

$$
\begin{gathered}
y_{k}=\frac{1}{T_{b}} \int r(t) c_{k}(t) d t \\
y_{k}=A_{k} b_{k}+\sum_{i=1}^{K} \rho_{i, k} A_{i} b_{i}+n_{k}(t)
\end{gathered}
$$

\section{MAI}

Outputs of the correlators can be stacked into a vector $y=\left[y_{1}\right.$. $\left.\ldots y_{K}\right]^{T}$, which can be expressed in matrix form as [9]

(4)

$$
\mathrm{y}=\mathrm{C} * \mathrm{~A} * \mathrm{~b}+\mathrm{n}
$$

Where

$C$ is a matrix that contains code correlations, $A$ is a diagonal matrix consisting of amplitudes and $b=\left[b_{1} \ldots b_{K}\right]^{T}$.

Decorrelating detector completely eliminates MAI (similar to zero-forcing equalizer in eliminating ISI). This is done by multiplying the correlator outputs by the inverse of the code correlation

$$
\mathrm{Z}_{\mathrm{DD}}=\mathrm{C}^{-1} \mathrm{y}=\mathrm{Ab}+\mathrm{C}^{-1} \mathrm{n}
$$

Hence, decorrelating detector successfully decouples all users. The limitation is that the noise increased, since elements of $C^{-1} n$ are always greater than or equal to the elements of the $n$ and another disadvantage is the computation of the inverse of 
the matrix. SIC takes a serial approach to canceling out interference. Each stage of the detector makes bit decisions, regenerates the signal and cancels it out so that it does not appear at the input of the next stage. The $i^{\text {th }}$ stage formulation is given below

$$
r^{(i+1)}(t)=r^{(i)}(t)-A_{k}(t) b_{k}(t) c_{k}(t)
$$

The amplitude estimates (in asynchronous systems also timing estimates) are required and hard decisions are performed for the symbols $b_{k}$. The canceller typically starts from the strongest signal because the probability of error is then smallest. The drawback is that each stage imposes an additional detection delay. Also, the detector suffers from error propagation and an error in the first stages causes erroneous symbol decisions also in later stages.

\section{SIMULATION ALGORITHM}

Multi-user detection (MUD) techniques also called joint detection and IC reduce the effects of multiple access interference and increase system capacity. SIC has also acted as a multiuser detection technique. The various SIC techniques are discussed in this paper but low complexity ZFSIC is used for multiuser detection or MAI suppression.

The simulation algorithm for multiuser detection consists of following steps

Step 1 Initially generates the random data of $10^{5}$ and PN sequence.

Step 2 Random data PN sequence spreading for wide band using XOR operation.

Step 3 The spreaded signal encode with convolution channel encoder (rate- 2/3) to make signal more specific.

Step 4 The encoded signal is modulated with QPSK and BPSK modulation technique.

Step 5 The modulated signal is processed through an RRC filter to make a signal band limited.

Step 6 White Gaussian noise varying signal to noise ratio (SNR) has been added to signal and the signal is transmitted through the AWGN channel.

Step 7 At receiver end since the signal is degraded with ISI, adjacent channel and co-channel interference, so the ZFSIC scheme has been applied to reduce the effect of interference. The ZF-SIC decoding algorithm consists of three parts [10].

a. To determine the transmitted stream with lowest error.

b. Estimation of the strongest transmitted signal by nulling out all weaker signals.

c. Interference cancellation: Demodulate the data bits, subtract their contribution from the received signal vector and return to the ordering step

Step 8 The signal is then processed through RRC filter and demodulated through QPSK and BPSK modulation schemes.

Step 9 The demodulated signal is decoded with viterbi decoder and the decoded signal is despreaded using XOR operation.

Step 10 The performance of the system is evaluated by comparing the degraded signal to the input signal and calculate the BER.

The flow of the simulated WCDMA transmitter and receiver system with ZF-SIC shown in Figure 1.

\section{SIMULATION RESULTS}

The simulation results with the number of users of 4, 6,9 and 15 under the AWGN in Figure 2- Figure 9. The work here investigates the impact of the number of users using ZF-SIC technique for WCDMA. The bit error rate (BER) performance is evaluated with varying conditions of signal to noise $(\mathrm{Eb} / \mathrm{No})$. The simulation analysis has been carried out for QPSK and BPSK. modulation in two cases. Case 1 is based on QPSK for ZF-SIC and case 2 is based on BPSK for ZF-SIC.

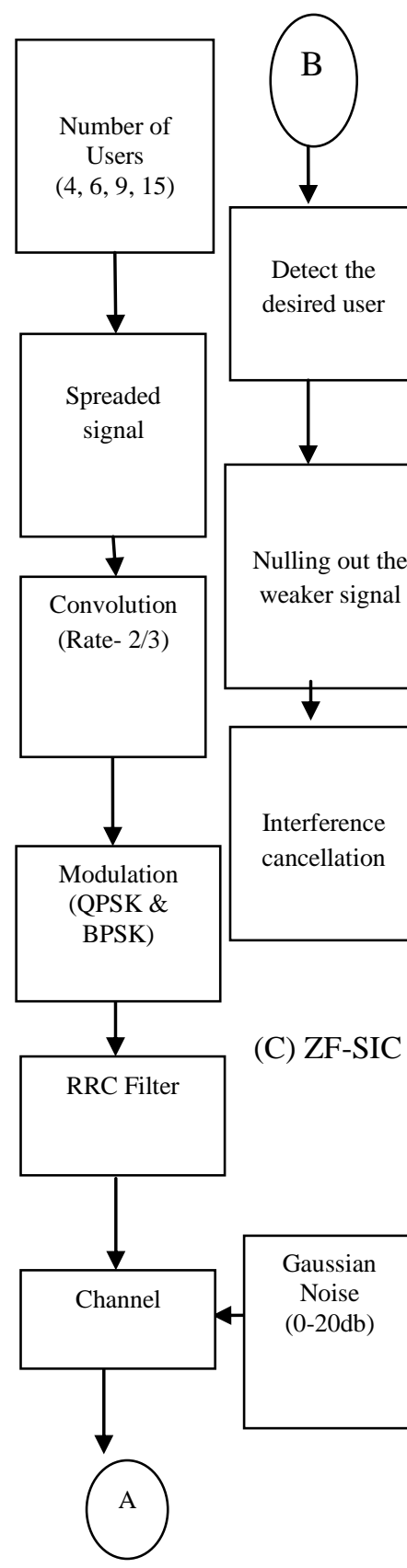

(a)
Transmitter

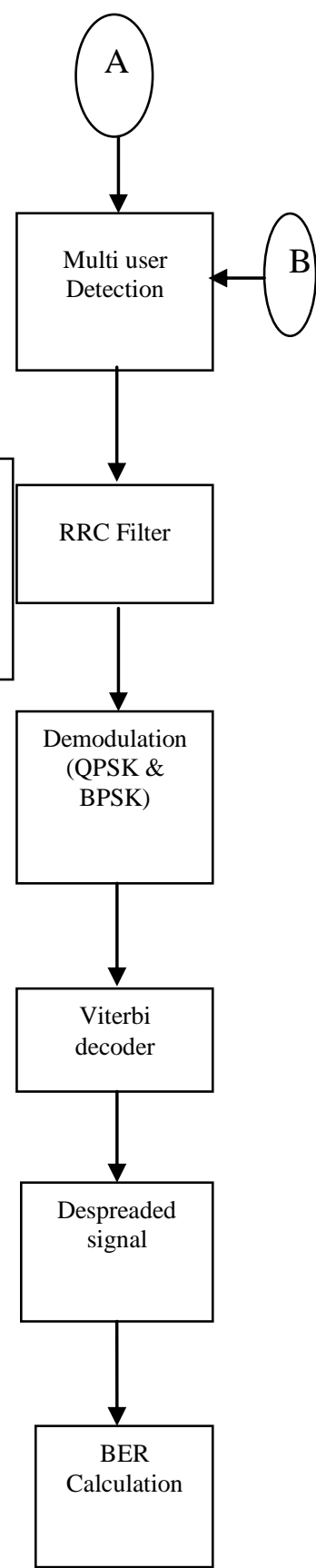

(b)

Receiver

Figure 1 Conceptual diagram of proposed WCDMA 


\subsection{BER Analysis of QPSK with ZF-SIC For Number of Users}

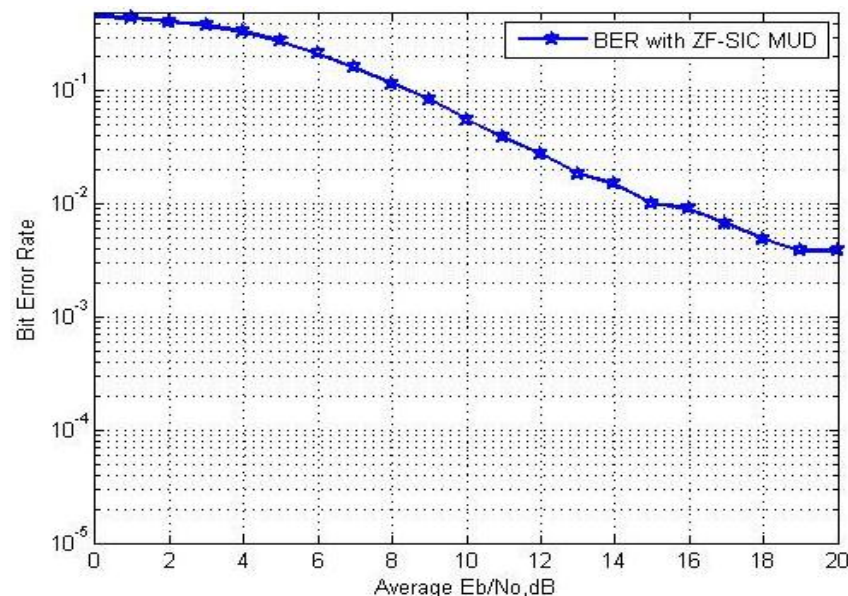

Figure 2 BER analysis of QPSK with ZF-SIC for 4 users.

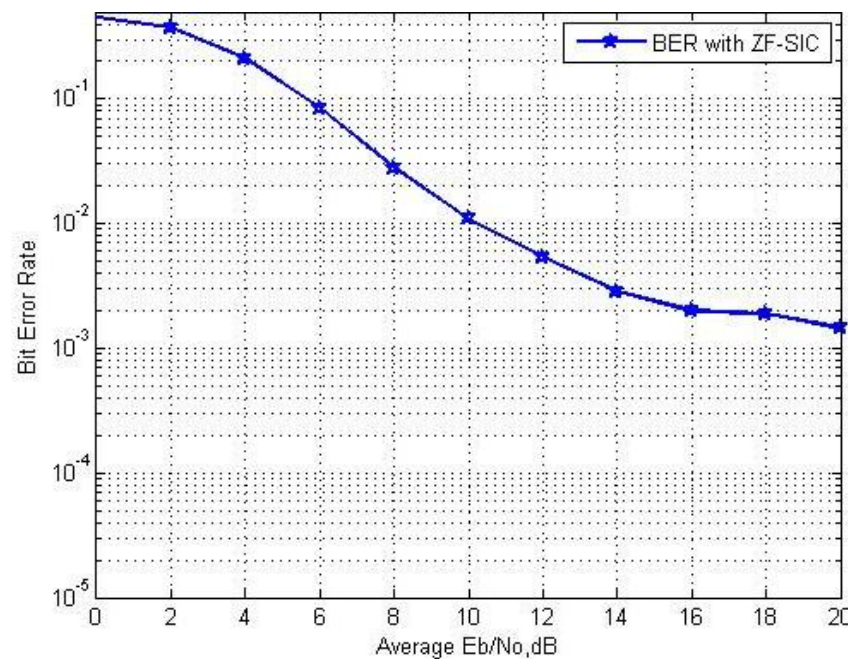

Figure 3 BER analysis of QPSK with ZF-SIC for 6 users

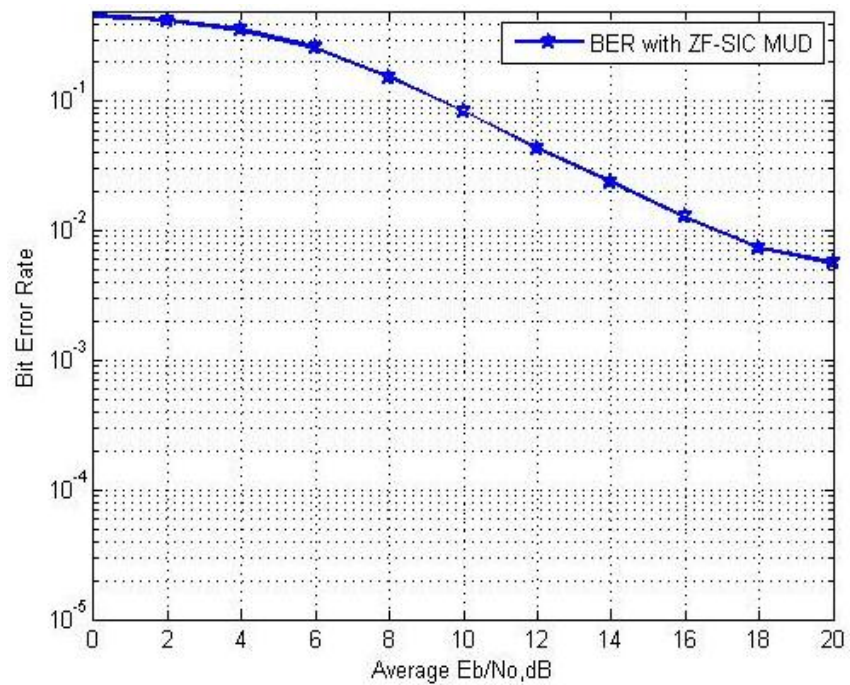

Figure 4 BER analysis of QPSK with ZF-SIC for 9 users

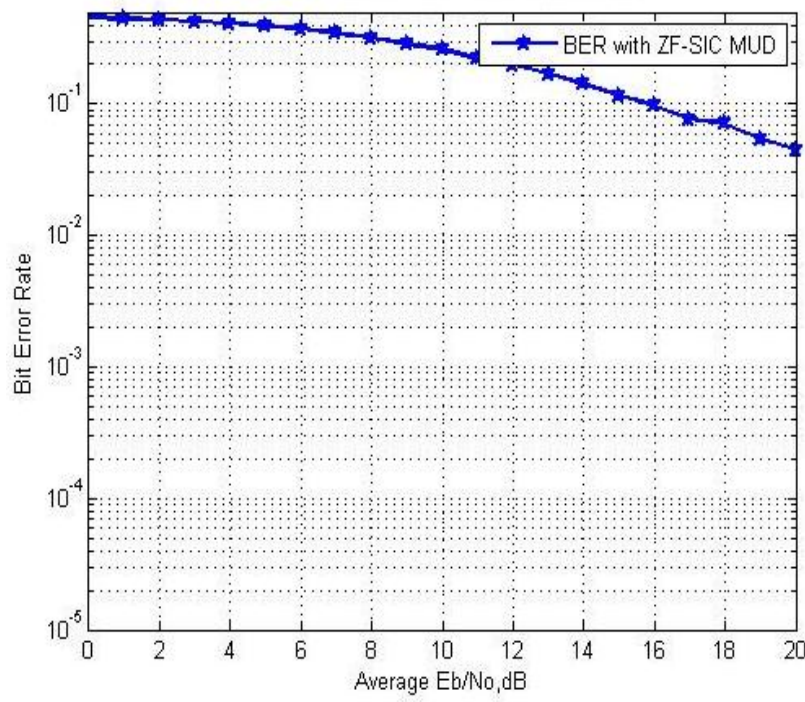

Figure 5 BER analysis of QPSK with ZF-SIC for 15 users

Discussion: - The analysis of BER v/s Eb/No for number of users with ZF-SIC is shown in Figure 2 to Figure 5. As can be seen from Figure 2 initially the BER obtained around 0.4550 and at higher values of Eb/No, the achievable BER decreases around 0.0014 for $18 \mathrm{~dB} \mathrm{~Eb} / \mathrm{No}$ with 4 users. With 6 users initially the BER obtained around 0.4510 and at higher values of Eb/No, the achievable BER decreases around 0.0034 for 20 $\mathrm{dB}$ Eb/No shown in Figure 3 From Figure 4 initially the BER obtained around 0.4589 and at higher values of $\mathrm{Eb} / \mathrm{No}$, the achievable BER decreases around 0.0057 for $20 \mathrm{~dB}$ Eb/No with 9 users. With 15 users initially the BER obtained around 0.5040 and at higher values of $\mathrm{Eb} / \mathrm{No}$, the achievable BER decreases around 0.0440 for $20 \mathrm{~dB}$ Eb/No shown in Figure 5 . It was observed from the simulation that the BER is gradually increased when the number of users increased.

\subsection{BER Analysis of BPSK with ZF-SIC}

\section{For Number of Users}

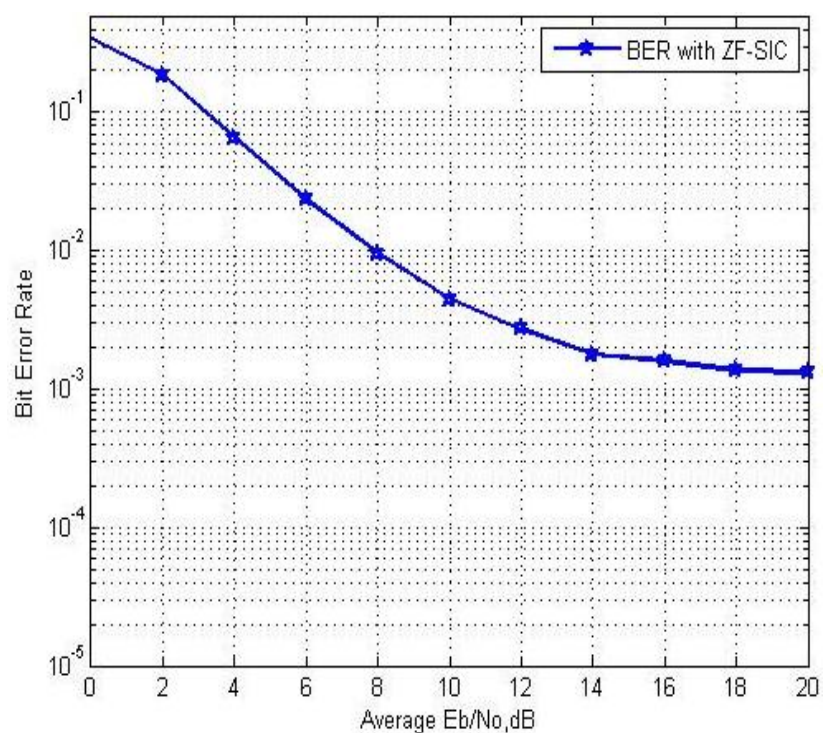

Figure 6 BER analysis of BPSK with ZF-SIC for 4 users 


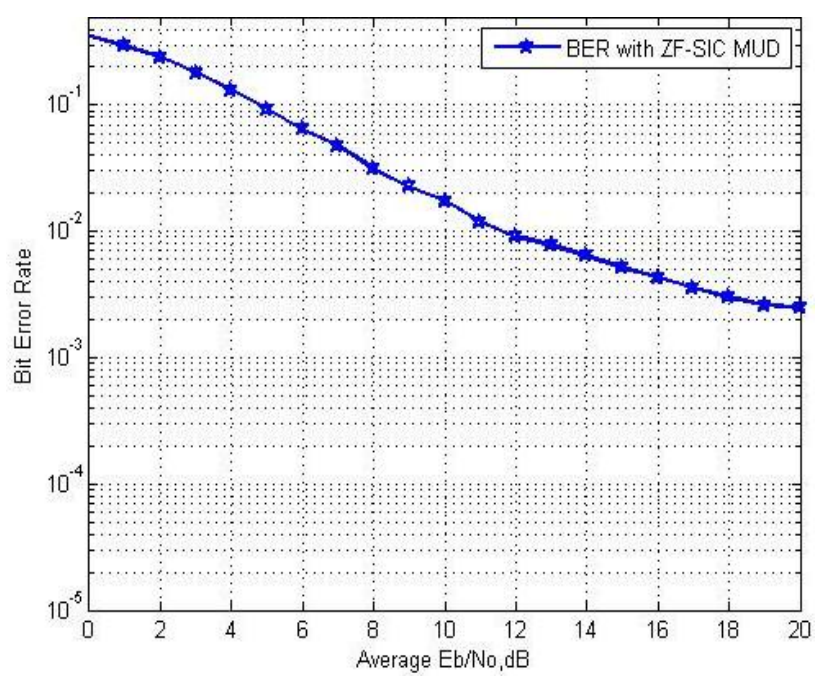

Figure 7 BER analysis of BPSK with ZF-SIC for 6 users

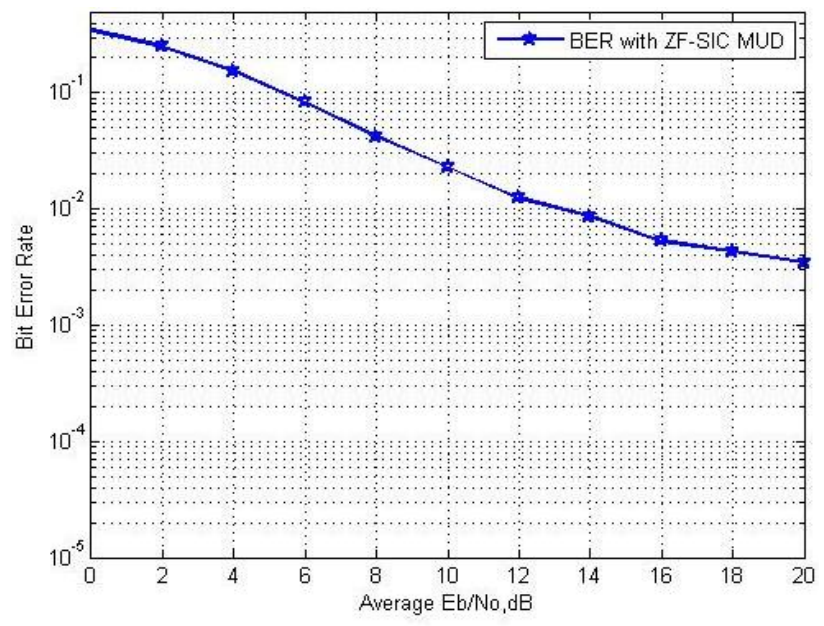

Figure 8 BER analysis of BPSK with ZF-SIC for 9 users

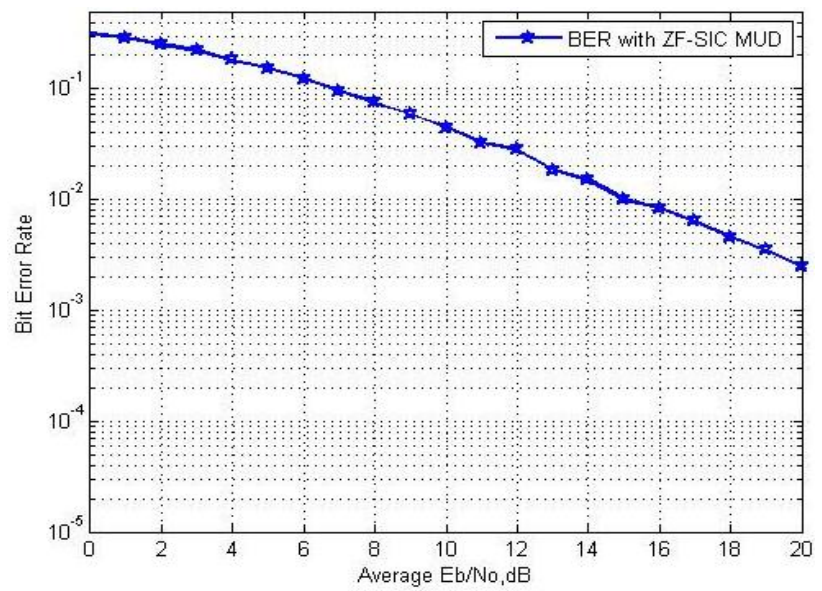

Figure 9 BER analysis of BPSK with ZF-SIC for 15 users

Discussion: - It was observed from the simulation results presented in Figure 6- Figure 9 that the BER gradually increase when the number of users increase from 4 to 15 . Initially a number of users are 4, the BER is less and it is around 0.3465 at $0 \mathrm{~dB}$ Eb/No and 0.0014 at $18 \mathrm{~dB}$ Eb/No. For a number of users 6 the BER is around 0.3472 at $0 \mathrm{~dB} \mathrm{~Eb} / \mathrm{No}$ and 0.0026 at $18 \mathrm{~dB} \mathrm{~Eb} / \mathrm{No}$. Similarly the BER is increased for 9 and 15 users and it is observed from the simulation results are 0.0039 and 0.0070 at $20 \mathrm{~dB} \mathrm{~Eb} / \mathrm{No}$. It is concluded the simulation that the BER was gradually increased when the number of users increased.

\section{CONCLUSION}

The work present in this paper analyzed the BER performance with number of users. The analysis was carried out for $4,6,9$ and 15 users. The simulation results showed that the BER performance decreased when the number of users increased for both BPSK and QPSK modulation with ZF-SIC. The ZFSIC scheme reduced the MAI but as the number of users increased the detection of strongest signal is more typical and the BER was decreased as observed in simulation results. It was found from the simulation results that the ZF-SIC scheme performed better BER for BPSK modulation, since the interference was reduced and system performance was increased.

\section{REFERENCES}

[1] Holma, H. and Toskala, A.2002 WCDMA for UMTS, John Wiley \& Sons Ltd., $1^{\text {st }}$ edition

[2] B. P. Lathi 1991 Modern digital and analog communications systems, John Wiley \& Sons, $2^{\text {nd }}$ edition.

[3] John G. Proakis, Digital Communications. McGraw Hill.

[4] M. Suryanegara, E.R. Hutabarat and D. Gunawan, 2006 "The interference On WCDMA system In 3G Coexistence Network.8" The 17th Annual IEEE International Symposium on Personal, Indoor and Mobile Radio Communications, pp. 1-5.

[5] Kim,D.J.; Sung,J. K.; Sung,R. K.; Chang, E. K., 1998 "Multiuser Detection using Blockwise Successive Interference Cancellation in DS/CDMA Systems", in Proceedings of IEEE International Conference on Vehicular Technology, Ottawa, Canada, Vol.3, pp.18351838 .

[6] Verdu, S. 1986 "Minimum Probability of Error for Asynchronous Multiple Acess Communication Systems", IEEE Transactions on Information Theory, Vol. 32, No. 1 , pp 85-96.

[7] Fang, L., and Milstein L. B. 2000 "Successive Interference Cancellation in Multicarrier DS/CDMA", IEEE Transactions on Communications, Vol. 48, No.2, pp. $1530-1540$.

[8] Andrews, J. G. and Teresa, H. Y. M., 2004 "Performance of Multicarrier CDMA With Successive Interference Cancellation in a Multipath Fading Channel", IEEE Transactions on Communications, Vol.52, No. 5, pp. 796-807.

[9] Moshavi,S. 1996 "Multi-user Detection for DS-CDMA Communications",IEEE Communications Magazine Vol.34, No.10, pp.124-136.

[10] Kumar, N.S. and Kumar, K.R.S. 2011 "Performance analysis and comparison of zero -forcing SIC and MMSE SIC for MIMO receivers using BSPK and 16- QAM modulation methods", International journal of computer science engineering and technology, Vol. 1, No. 8, pp. 530-533. 